PROCEEDINGS OF THE

AMERICAN MATHEMATICAL SOCIETY

Volume 134, Number 4, Pages 959-972

S 0002-9939(05)08041-X

Article electronically published on August 16, 2005

\title{
THE EQUIVARIANT BRAUER GROUP OF A GROUP
}

\author{
S. CAENEPEEL, F. VAN OYSTAEYEN, AND Y. H. ZHANG
}

(Communicated by Martin Lorenz)

\begin{abstract}
We consider the Brauer group $\operatorname{BM}^{\prime}(k, G)$ of a group $G$ (finite or infinite) over a commutative ring $k$ with identity. A split exact sequence

$$
1 \longrightarrow \operatorname{Br}^{\prime}(k) \longrightarrow \operatorname{BM}^{\prime}(k, G) \longrightarrow \operatorname{Gal}(k, G) \longrightarrow 1
$$

is obtained. This generalizes the Fröhlich-Wall exact sequence from the case of a field to the case of a commutative ring, and generalizes the Picco-Platzeck exact sequence from the finite case of $G$ to the infinite case of $G$. Here $\operatorname{Br}^{\prime}(k)$ is the Brauer-Taylor group of Azumaya algebras (not necessarily with unit). The method developed in this paper might provide a key to computing the equivariant Brauer group of an infinite quantum group.
\end{abstract}

\section{INTRODUCTION}

In [9, 10, Fröhlich and Wall introduced the equivariant Brauer group $\operatorname{BM}(k, G)$ of a finite group $G$ and a commutative ring $k$, consisting of equivalence classes of $k$-Azumaya algebras on which $G$ acts as a group of automorphisms. In 8] Fröhlich studied further the equivariant Brauer group of an arbitrary group over a field. $\operatorname{BM}(k, G)$ can be completely described by the Brauer group $\operatorname{Br}(k)$ and the second cohomology group $H^{2}(G, U(k))$ via the following split exact sequence when $\operatorname{Pic}(k)=1$ :

$$
1 \rightarrow \operatorname{Br}(k) \rightarrow \operatorname{BM}(k, G) \rightarrow H^{2}(G, U(k)) \rightarrow 1 .
$$

If $\operatorname{Pic}(k) \neq 1$, then we replace $H^{2}(G, U(k))$ by the $\operatorname{group} \operatorname{Gal}(k, G)$ of $k G$-Galois objects (these are $G$-strongly graded rings having $k$ as part of degree $e$ ). This sequence is originally due to Picco and Platzeck [14], and several variations and generalizations of it have appeared in the literature. Beattie [1 generalized the sequence to the Brauer group of algebras with an action by a finitely generated projective cocommutative Hopf algebra, and Childs 7] gave an exact sequence involving algebras with a finite abelian group grading inducing a coaction, using a bilinear map. All the generalizations (see [2] for a detailed discussion) have in common that the (co)acting group or Hopf algebra is finite.

The aim of this note is to extend the Picco-Platzeck exact sequence to infinite groups. We can still introduce the invariants $\operatorname{Gal}(k, G), H^{2}(G, U(k))$ and $\operatorname{BM}(k, G)$, and define a map $\pi: \operatorname{BM}(k, G) \rightarrow \operatorname{Gal}(k, G)$, but the problem is that $\pi$ is in general

Received by the editors December 16, 2003 and, in revised form, August 16, 2004 and November 1,2004 .

2000 Mathematics Subject Classification. Primary 16H05, 16W50.

Key words and phrases. Equivariant Brauer group, Taylor Azumaya algebra.

The third named author was supported by the Marsden Fund.

(C)2005 American Mathematical Society 
not surjective if $G$ is infinite. The idea is then to replace the Brauer group by the Brauer-Taylor group. Taylor [16] defined the Brauer-Taylor group $\operatorname{Br}^{\prime}(k)$, consisting of equivalence classes of Azumaya algebras without a unit. If $k$ is noetherian, then there is an isomorphism between $\operatorname{Br}^{\prime}(k)$ and the second étale cohomology group. It is known that the second étale cohomology group, and, a fortiori, Taylor's Brauer group is not always torsion, and it follows from Gabber's Theorem [11, 12, that the classical Brauer group $\operatorname{Br}(k)$ is exactly the torsion part of $\operatorname{Br}^{\prime}(k)$.

Let $G$ be a group, and consider Taylor-Azumaya algebras on which $G$ acts as a group of automorphisms. Such a Taylor-Azumaya algebra is called a $G$-module Taylor-Azumaya algebra. The set of isomorphism classes of $G$-module TaylorAzumaya algebras modulo Morita equivalence is a group, called the equivariant Brauer-Taylor group of $G$ and $k$.

In Sections 1.1 and 1.2 we recall some facts about the Brauer-Taylor group and about the group of Galois objects. Our methods will be based on the theory of multiplier algebras and multiplier Hopf algebras, and these are briefly discussed in Sections 1.3 and 1.4. The equivariant Brauer-Taylor group is defined in Section 1.5. In Section 2 we define the group homomorphism $\pi$ from the Taylor-Brauer group to the group of $k G$-Galois objects and show that $\pi$ is surjective and split. The main result is the exact sequence in Theorem 2.12, describing the equivariant BrauerTaylor group as the direct sum of the Brauer-Taylor group and the group of $k G$ Galois objects.

We will use the following notation: if $C$ is an object of a category, then $C$ will also be a notation for the identity morphism on $C$.

\section{Preliminaries}

1.1. Taylor-Azumaya algebras. In [16], Taylor introduced a Brauer group consisting of equivalence classes of Azumaya algebras without a unit. This new invariant was investigated further in [4, 15]. Our main reference will be [2].

Let $k$ be a commutative ring, and $A$ a $k$-algebra, not necessarily with unit. We call $A$ a unital $k$-algebra if the canonical morphism $A \otimes_{A} A \rightarrow A$ is an isomorphism. In a similar way, a left $A$-module $M$ is called unital if the canonical map $A \otimes_{A} M \rightarrow$ $M$ is an isomorphism. An algebra with unit is unital, and then any module is unital. If $A$ is a unital algebra, then ${ }_{A} \mathcal{M}^{u}$ will be the category of unital left $A$-modules. Unital right modules and bimodules are defined in a similar way.

If $A$ is a unital $k$-algebra, then the enveloping algebra $A^{\mathrm{e}}=A \otimes A^{\mathrm{op}}$ is also unital. The center of $A$ is defined as $Z(A)=\operatorname{End}_{A^{\mathrm{e}}}(A)$. If $A$ has a unit, then we recover the classical definition of the center. From the fact that $A^{2}=A$, it follows that $Z(A)$ is a commutative $k$-algebra with unit, and $A$ is a $Z(A)$-algebra. $A_{l}$ (resp. $A_{r}$ ) will be our notation for $A$ when viewed as a left (resp. a right) $A^{\mathrm{e}}$-module. We can define (strict) Morita contexts connecting unital algebras in the obvious way: we require that the connecting bimodules are unital bimodules (see 2, Sec. 1.1]).

Definition 1.1. Let $A$ be a unital, faithful $k$-algebra. $A$ is called a Taylor-Azumaya algebra if it satisfies the following equivalent conditions.

(1) There exists an invertible $k$-module $I$ such that the functors

$$
\begin{aligned}
F:{ }_{k} \mathcal{M} & \rightarrow{ }_{A^{\mathrm{e}}} \mathcal{M}^{u}, F(N)=A_{l} \otimes N \text { and } \\
H: A^{\mathrm{e}} \mathcal{M}^{u} & \rightarrow{ }_{k} \mathcal{M}, H(M)=\left(A_{r} \otimes I\right) \otimes A^{\mathrm{e}} M
\end{aligned}
$$

form a pair of inverse equivalences; 
(2) the pair of adjoint functors $\left(F, H_{l}\right)$, with

$$
H_{l}:{ }_{A^{\mathrm{e}}} \mathcal{M}^{u} \rightarrow{ }_{k} \mathcal{M}, H_{l}(M)=\operatorname{Hom}_{A^{\mathrm{e}}}\left(A_{l}, M\right)
$$

is a pair of inverse equivalences.

For the proof of the equivalence of the conditions, we refer to [2, Prop. 2.2.5 and 2.2.8]. From the second condition, it follows immediately that a Taylor-Azumaya algebra is central. A Taylor-Azumaya algebra with a unit is a usual Azumaya algebra. More conditions characterizing Taylor-Azumaya algebras may be found in 2. Sec. 2.2, 4.2 and 4.3]. If $A$ is a Taylor-Azumaya algebra, then the functors $H$ and $H_{l}$ from the definition are isomorphic.

Consider two $k$-modules $M$ and $M^{\prime}$ and a surjective $k$-linear map $\mu: M^{\prime} \otimes M \rightarrow$ $k$. We call $\underline{M}=\left(M, M^{\prime}, \mu\right)$ a dual pair of $k$-modules, and define the associated elementary algebra $\mathrm{E}(\underline{M})=M \otimes M^{\prime}$, with multiplication

$$
\left(m \otimes m^{\prime}\right)\left(n \otimes n^{\prime}\right)=\mu\left(m^{\prime} \otimes n\right) m \otimes n^{\prime},
$$

for $m, n \in M$ and $m^{\prime}, n^{\prime} \in M^{\prime}$. Then $\mathrm{E}(\underline{M})$ is a Taylor-Azumaya algebra. $M$ is a unital left $\mathrm{E}(\underline{M})$-module, and $M^{\prime}$ is a unital right $\mathrm{E}(\underline{M})$-module; the actions are given by the formulas

$$
\left(m \otimes m^{\prime}\right) n=m \mu\left(m^{\prime} \otimes n\right) \text { and } n^{\prime}\left(m \otimes m^{\prime}\right)=\mu\left(n^{\prime} \otimes m\right) m^{\prime} .
$$

If $\mathrm{E}(\underline{M})$ has a unit, then $M$ and $M^{\prime}$ are faithfully projective as $k$-modules, $M^{\prime} \cong$ $M^{*}$, and $\mu: M^{\prime} \otimes M \cong M^{*} \otimes M \rightarrow k$ is the evaluation map. In this case, $\mathrm{E}(\underline{M}) \cong \operatorname{End}_{k}(M)$.

Proposition 1.2 (2, Prop. 3.1.1]). Let $A$ and $B$ be Taylor-Azumaya algebras. The following assertions are equivalent.

(1) $A$ and $B$ are Morita equivalent;

(2) $A \otimes B^{\mathrm{op}}$ is an elementary algebra;

(3) there exist dual pairs $\underline{M}$ and $\underline{N}$ such that $A \otimes \mathrm{E}(\underline{M}) \cong B \otimes \mathrm{E}(\underline{N})$ as $k$ algebras.

Now consider the set $\operatorname{Br}^{\prime}(k)$ of Morita equivalence classes represented by a finitely generated Taylor-Azumaya algebra. $\operatorname{Br}^{\prime}(k)$ is a group under the operation induced by the tensor product (see [2, Sec. 3.2]). We recall from [4 that every flat TaylorAzumaya algebra is Morita equivalent to a finitely generated Taylor-Azumaya algebra. There exist examples of Taylor-Azumaya algebras that are not flat (see [4] or [2, Sec. 4.3]). If $k$ is a field, then $\operatorname{Br}^{\prime}(k)=\operatorname{Br}(k)$.

1.2. Strongly graded rings. Let $k$ be a commutative ring, and $G$ an arbitrary group. A $G$-graded $k$-algebra $S$ is called a $k G$-Galois object if the canonical map

$$
\gamma: S \otimes S \rightarrow S \otimes k G, \gamma(s \otimes t)=\sum_{g \in G} s t_{g} \otimes g
$$

is an isomorphism. This is equivalent to (see [2, Prop. 8.2.1])

- $S$ is strongly graded, that is, $S_{g} S_{h}=S_{g h}$, for all $g, h \in G$;

- $S_{e}=k$, where $e$ is the unit element of $G$. 
The set of equivalence classes $\operatorname{Gal}(k, G)$ of $k G$-Galois objects forms a group under the operation induced by the cotensor product:

$$
S \square T=\bigoplus_{g \in G} S_{g} \otimes T_{g} .
$$

1.3. Multipliers. From [17, we recall the notion of multiplier algebra of an algebra $A$. A multiplier of $A$ is a pair $\left(\rho_{1}, \rho_{2}\right)$ consisting of a right $A$-linear map $\rho_{1}$ and a left $A$-linear map $\rho_{2}$ such that $a \rho_{1}(b)=\rho_{2}(a) b$ for all $a, b \in A$. If $x=\left(\rho_{1}, \rho_{2}\right)$ is a multiplier, then we write $x b=\rho_{1}(b)$ and $b x=\rho_{2}(b) . M(A)$, the set of all multipliers on $A$, is a $k$-algebra with multiplication $\left(\rho_{1}, \rho_{2}\right)\left(\rho_{1}^{\prime}, \rho_{2}^{\prime}\right)=\left(\rho_{1} \circ \rho_{1}^{\prime}, \rho_{2}^{\prime} \circ \rho_{2}\right)$ and unit $(A, A)$. We have an algebra map $A \rightarrow M(A)$; the multiplier corresponding to $a \in A$ is given by left and right multiplication. $M(A)$ is an algebra with unit $(A, A)$. If $A$ has identity, then $A=M(A)$. Indeed, if $x=\left(\rho_{1}, \rho_{2}\right) \in M(A)$, then $\rho_{1}(1)=1 \rho_{1}(1)=\rho_{2}(1) 1=\rho_{2}(1)$, and $\rho_{1}(b)=1 \rho_{1}(b)=\rho_{2}(1) b, \rho_{2}(c)=c \rho_{1}(1)$. Let us compute the multiplier algebra of an elementary algebra.

Proposition 1.3. Let $\underline{M}=\left(M, M^{\prime}, \mu\right)$ be a dual pair, and $E=E(\underline{M})$ the corresponding elementary algebra. Then the multiplier algebra $M(E)$ is isomorphic to

$\bar{E}=\left\{\left(f, f^{\prime}\right) \in E_{1} \times E_{2} \mid \mu\left(m^{\prime} \otimes f(m)\right)=\mu\left(f^{\prime}\left(m^{\prime}\right) \otimes m\right)\right.$, for all $\left.m \in M, m^{\prime} \in M^{\prime}\right\}$, where $E_{1}=\operatorname{End}(M)$ and $E_{2}=\operatorname{End}\left(M^{\prime}\right)^{o p}$.

Proof. We have a map

$$
\alpha: \bar{E} \rightarrow M(E), \alpha\left(f, f^{\prime}\right)=x=\left(\rho_{1}=f \otimes M^{\prime}, \rho_{2}=M \otimes f^{\prime}\right) .
$$

It is straightforward to see that $x$ is a multiplier.

Next we define

$$
\beta: M(E) \rightarrow \bar{E}, \beta\left(\rho_{1}, \rho_{2}\right)=\left(f, f^{\prime}\right),
$$

with $f: M \rightarrow M$ and $f^{\prime}: M^{\prime} \rightarrow M^{\prime}$ are defined as follows:

$$
f(m)=\sum_{i} \rho_{1}\left(m \otimes m_{i}^{\prime}\right) m_{i} \text { and } f^{\prime}\left(m^{\prime}\right)=\sum_{i} m_{i}^{\prime} \rho_{2}\left(m_{i} \otimes m^{\prime}\right),
$$

where $\mu\left(\sum_{i} m_{i}^{\prime} \otimes m_{i}\right)=1$. We compute that

$$
\begin{aligned}
& \rho_{1}\left(m \otimes m^{\prime}\right)=\sum_{i} \rho_{1}\left(\left(m \otimes m_{i}^{\prime}\right)\left(m_{i} \otimes m^{\prime}\right)\right)=\sum_{i} \rho_{1}\left(m \otimes m_{i}^{\prime}\right) m_{i} \otimes m^{\prime}, \\
& \rho_{2}\left(m \otimes m^{\prime}\right)=\sum_{i} \rho_{2}\left(\left(m \otimes m_{i}^{\prime}\right)\left(m_{i} \otimes m^{\prime}\right)\right)=\sum_{i} m \otimes m_{i}^{\prime} \rho_{1}\left(m_{i} \otimes m^{\prime}\right) .
\end{aligned}
$$

Since $\left(\rho_{1}, \rho_{2}\right)$ is a multiplier, we have, for all $m, n \in M$ and $m^{\prime}, n^{\prime} \in M^{\prime}$, that

$$
\begin{aligned}
& \left(m \otimes m^{\prime}\right) \rho_{1}\left(n \otimes n^{\prime}\right)=\mu\left(m^{\prime} \otimes f(n)\right) m \otimes n^{\prime} \\
& \quad=\rho_{2}\left(m \otimes m^{\prime}\right) n \otimes n^{\prime}=\mu\left(f^{\prime}(m) \otimes n\right) m \otimes n^{\prime},
\end{aligned}
$$

and it follows from the fact that $E$ is faithful that $\left(f, f^{\prime}\right) \in \bar{E}$. Finally, it is easy to see that $\alpha$ is the inverse of $\beta$. 
1.4. Multiplier Hopf algebras. From [17, we recall the definition of a multiplier Hopf algebra. If $A$ is a unital algebra, then $A \otimes A$ is again a unital algebra, with non-degenerate product. The tensor product $M(A) \otimes M(A)$ is in a natural way a subalgebra of $M(A \otimes A)$, and the embedding $A \rightarrow M(A \otimes A)$ factors through this map. A multiplier Hopf algebra is a unital algebra $A$ together with an algebra map $\Delta: A \otimes A \rightarrow M(A \otimes A)$ such that $\Delta(a)(1 \otimes b)$ and $(a \otimes 1) \Delta(b)$ belong to $A \otimes A$ for all $a, b \in A$ and $\Delta$ is coassociative in the sense that

$$
(a \otimes 1 \otimes 1)(\Delta \otimes A)(\Delta(b)(1 \otimes c))=(A \otimes \Delta)((a \otimes 1) \Delta(b))(1 \otimes 1 \otimes c)
$$

for all $a, b, c \in A$. In this paper, we will need the following example of multiplier Hopf algebra.

Example 1.4. Let $G$ be a group. Let $(k G)^{*}$ be the dual of the group algebra $k G$. Let $p_{g}: k G \rightarrow k$ be the map defined by $p_{g}(h)=\delta_{g, h}$, and $k(G)=\bigoplus_{g \in G} k p_{g}$. Then $M(k(G))=(k G)^{*}$. In a similar way, $M(k(G \times G))=(k G \times k G)^{*}$. There is a natural identification $k(G) \otimes k(G) \cong k(G \times G)$, and $M(k(G) \otimes k(G)) \cong M(k(G \times G))$. The multiplication on $G$ induces an algebra map $\Delta: k(G) \rightarrow M(k(G) \otimes k(G))$ given by $(\Delta(f))(p, q)=f(p q)$. One may use (informal) Sweedler sigma notation for the comultiplication

$$
\Delta\left(p_{g}\right)=\sum p_{g_{(1)}} \otimes p_{g_{(2)}}=\sum_{h \in G} p_{h} \otimes p_{h^{-1} g}
$$

for $p_{g} \in k(G)$. Moreover, we have that $\Delta\left(f_{1}\right)\left(1 \otimes f_{2}\right) \in k(G) \otimes k(G)$ and $\left(f_{1} \otimes 1\right) \Delta\left(f_{2}\right) \in k(G) \otimes k(G)$, for all $f_{1}, f_{2} \in k(G)$. From the associativity of the multiplication on $G$, it easily follows that $\Delta$ is coassociative. Thus $(k(G), \Delta)$ is a multiplier Hopf algebra.

1.5. The equivariant Brauer group. Let $A$ be a $k$-algebra and $G$ a group. If $G$ acts as a group of $k$-algebra automorphisms on $A$ with trivial $G$-action on $k$, then we say that $A$ is a $G$-module algebra. A Taylor-Azumaya algebra $A$ that is also a $G$ module algebra is called a $G$-module Taylor-Azumaya algebra. If $\underline{M}=\left(M, M^{\prime}, \mu\right)$ is a dual pair, such that $M$ and $M^{\prime}$ are $G$-modules, and

$$
\mu\left(g \cdot m^{\prime} \otimes g \cdot m\right)=\mu\left(m^{\prime} \otimes m\right),
$$

for all $g \in G, m \in M$ and $m^{\prime} \in M^{\prime}$, then $\mathrm{E}(\underline{M})$ is a $G$-module Taylor-Azumaya algebra, with diagonal action $g \cdot\left(m \otimes m^{\prime}\right)=g \cdot m \otimes g \cdot m^{\prime}$. We say that $\mathrm{E}(\underline{M})$ is an elementary $G$-module algebra.

Let $A$ and $B$ be unital $G$-module algebras. We can define (strict) Morita contexts connecting $A$ and $B$, requiring that the bimodules in the Morita contexts are $G$ modules that are unital on both sides, and that the connecting bimodule maps preserve the $G$-action. We have the following generalization of Proposition 1.2 , The proof is an easy adaption of the proof of [2, Prop. 3.1.1].

Proposition 1.5. Let $A$ and $B$ be $G$-module Taylor-Azumaya algebras. The following assertions are equivalent:

(1) $A$ and $B$ are Morita equivalent as $G$-module algebras;

(2) $A \otimes B^{\text {op }}$ is an elementary $G$-module algebra;

(3) there exist dual pairs of $G$-modules $\underline{M}$ and $\underline{N}$ such that $A \otimes \mathrm{E}(\underline{M}) \cong B \otimes$ $\mathrm{E}(\underline{N})$ as $G$-module algebras. 
Now we introduce the equivariant Brauer group $\operatorname{BM}^{\prime}(k, G)$ as the set of Morita equivalence classes of $G$-module Taylor-Azumaya algebras, represented by a finitely generated $G$-module Taylor-Azumaya algebra. It is a group under the operation induced by the tensor product. If $k$ is a field, then $\operatorname{BM}^{\prime}(k, G) \cong \operatorname{BM}(k, G)$, the Brauer group of $G$-module Azumaya algebras.

\section{The SPlit EXaCt SEQuence}

Let $k$ be a commutative ring, $A$ a unital $k$-algebra, and $G$ a group. ${ }_{k} \mathcal{M}^{G}$ will be the category of graded $k$-modules, and ${ }_{A} \mathcal{M}^{u G}$ the category of $G$-graded unital left $A$-modules. Take an invertible $k$-module $I$. The functors $F$ and $H$ from Definition 1.1 send graded modules to graded modules. If $A$ is a Taylor-Azumaya algebra, then $H \cong H_{l}$, hence $H_{l}$ also sends graded modules to graded modules, and we have a pair of inverse equivalences $\left(F, H_{l}\right)$, with

$$
F:{ }_{k} \mathcal{M}^{G} \rightarrow{ }_{A^{\mathrm{e}}} \mathcal{M}^{u G}, F(N)=A_{l} \otimes N
$$

and

$$
H_{l}:{ }_{A^{\mathrm{e}}} \mathcal{M}^{u G} \rightarrow{ }_{k} \mathcal{M}^{G}, H_{l}(M)=\operatorname{Hom}_{A^{\mathrm{e}}}(A, M) .
$$

In particular, if $M$ is a $G$-graded unital left $A^{\mathrm{e}}$-module, then $\operatorname{Hom}_{A^{\mathrm{e}}}(A, M)$ is also $G$-graded. This means that every left $A^{\mathrm{e}}$-linear map $f: A \rightarrow M$ can be decomposed into a finite sum $f=\sum_{g \in G} f_{g}$, with $f_{g}(A) \subset M_{g}$, for all $g$.

Let $A$ be a $G$-module algebra, and consider the smash product $S_{A}=A \# k G$, with multiplication

$$
(a \# g)(b \# h)=a(g \cdot b) \# g h,
$$

for $a, b \in A$ and $g, h \in G$. We have an algebra map $\eta: A \rightarrow S_{A}, \eta(a)=a \# 1_{G}$, making $A$ into a subalgebra of $S_{A}$. If $A$ is a unital algebra, then $S_{A}$ is a $G$-graded unital left $A^{\mathrm{e}}$-module, with left action $\left(a \otimes a^{\prime}\right) \cdot(b \# g)=a b\left(g \cdot a^{\prime}\right) \# g$, and with $G$-grading given by $\operatorname{deg}(b \# g)=g$.

Lemma 2.1. Let $A$ be a $G$-module Taylor-Azumaya algebra. Then $\operatorname{Hom}_{A^{\mathrm{e}}}\left(A, S_{A}\right)$ $=H_{l}\left(S_{A}\right)$ is a $k G$-Galois object.

Proof. We have already seen at the beginning of this section that $H_{l}(M)$ is a $G$-graded module, for every $G$-graded unital left $A^{\mathrm{e}}$-module $M$. Take $\varphi, \psi \in$ $\operatorname{Hom}_{A^{\mathrm{e}}}\left(A, S_{A}\right)$. The product of $\varphi$ and $\psi$ is defined as follows: for all $a \in A$, take $a_{i}, a_{i}^{\prime} \in A$ such that $a=\sum_{i} a_{i} a_{i}^{\prime}$, and put

$$
(\varphi \psi)(a)=\sum_{i} \varphi\left(a_{i}\right) \psi\left(a_{i}^{\prime}\right) .
$$

The product is well-defined because $A \cong A \otimes_{A} A$, and both $\varphi$ and $\psi$ are $A$ bimodule maps. It is easy to see that $\eta$ is a unit for this multiplication, and that $\operatorname{Hom}_{A^{\mathrm{e}}}\left(A, S_{A}\right)$ is a $G$-graded algebra. It remains to be shown that the canonical map

$\gamma: \operatorname{Hom}_{A^{\mathrm{e}}}\left(A, S_{A}\right) \otimes \operatorname{Hom}_{A^{\mathrm{e}}}\left(A, S_{A}\right) \rightarrow \operatorname{Hom}_{A^{\mathrm{e}}}\left(A, S_{A}\right) \otimes k G, \gamma(\varphi \otimes \psi)=\varphi \psi \otimes h$,

is an isomorphism if $\psi$ is homogeneous of degree $h$. Since $\left(F, H_{l}\right)$ is an equivalence of categories, it suffices to show that $F(\gamma)=A \otimes \gamma$ is an isomorphism. Since $\left(F, H_{l}\right)$ is an equivalence of categories, the evaluation map

$$
\text { ev }: A \otimes \operatorname{Hom}_{A^{\mathrm{e}}}\left(A, S_{A}\right) \rightarrow S_{A}, \operatorname{ev}(a \otimes \varphi)=\varphi(a)
$$


is an isomorphism. Hence we have isomorphisms

$$
\begin{gathered}
A \otimes \operatorname{Hom}_{A^{\mathrm{e}}}\left(A, S_{A}\right) \otimes \operatorname{Hom}_{A^{\mathrm{e}}}\left(A, S_{A}\right) \cong S_{A} \otimes \operatorname{Hom}_{A^{\mathrm{e}}}\left(A, S_{A}\right) \\
\cong S_{A} \otimes_{A} A \otimes \operatorname{Hom}_{A^{\mathrm{e}}}\left(A, S_{A}\right) \cong S_{A} \otimes_{A} S_{A} .
\end{gathered}
$$

The composition of these isomorphisms is called $\alpha$, and is given by

$$
\alpha(a b \otimes \varphi \otimes \psi)=\varphi(a) \otimes_{A} \psi(b) .
$$

Also,

$$
\text { ev } \otimes k G: A \otimes \operatorname{Hom}_{A}\left(A, S_{A}\right) \otimes k G \rightarrow S_{A} \otimes k G
$$

is an isomorphism, and we define $\beta$ by the commutativity of the following diagram:

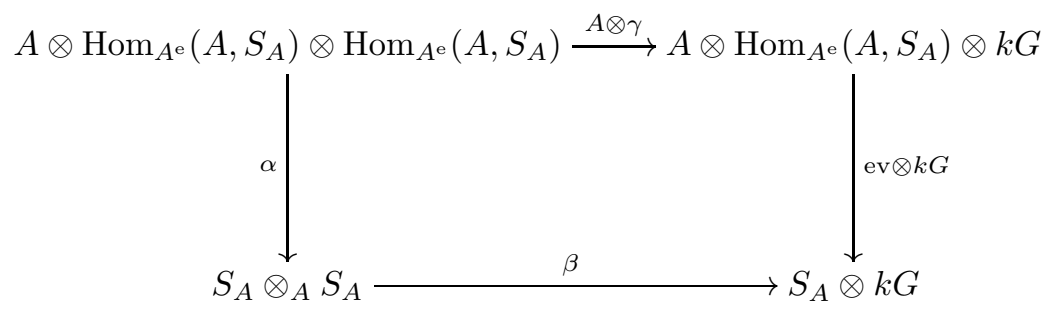

It now suffices to show that $\beta$ is an isomorphism. Take $a \# g, b \# h \in S_{A}$, and write $\mathrm{ev}^{-1}(a \# g)=\sum_{i} a_{i} \otimes \varphi_{i}, \mathrm{ev}^{-1}(b \# h)=\sum_{j} b_{j} \otimes \psi_{j}$, with $\varphi_{i}$ and $\psi_{j}$ homogeneous of respectively degree $g$ and $h$. Then

$$
\alpha\left(\sum_{i, j} a_{i} b_{j} \otimes \varphi_{i} \otimes \psi_{j}\right)=\sum_{i, j} \varphi_{i}\left(a_{i}\right) \otimes \psi_{j}\left(b_{j}\right)=(a \# g) \otimes_{A}(b \# h),
$$

hence

$$
\begin{aligned}
\beta((a \# g) & \left.\otimes_{A}(b \# h)\right)=(\mathrm{ev} \otimes k G)(A \otimes \gamma)\left(\sum_{i, j} a_{i} b_{j} \otimes \varphi_{i} \otimes \psi_{j}\right) \\
& =(\mathrm{ev} \otimes k G)\left(\sum_{i, j} a_{i} b_{j} \otimes \varphi_{i} \psi_{j} \otimes h\right)=\sum_{i, j}\left(\varphi_{i} \psi_{j}\right)\left(a_{i} b_{j}\right) \otimes h \\
& =\left(\sum_{i} \varphi_{i}\left(a_{i}\right)\right)\left(\sum_{j} \varphi_{j}\left(b_{j}\right)\right)=(a \# g)(b \# h)=a(g \cdot b) \# g h .
\end{aligned}
$$

A straightforward computation shows that the inverse of $\beta$ is given by the formula

$$
\beta^{-1}((a b \# g) \otimes h)=\left(a \# g h^{-1}\right) \otimes_{A}\left(h g^{-1}\right) \cdot b \# h .
$$

We will now present some alternative descriptions of $\operatorname{Hom}_{A^{\mathrm{e}}}(A, A \# k G)$. The first one is based on the fact that $\operatorname{Hom}_{A^{\mathrm{e}}}(A, A \# k G)$ is graded, and the second one is in terms of multipliers. Then we will show that there is an anti-algebra homomorphism to the multipliers of $A$.

Lemma 2.2. Let $A$ be a $G$-module Taylor-Azumaya algebra. The part of degree $g$ of $\operatorname{Hom}_{A^{\mathrm{e}}}(A, A \# k G)$ is isomorphic to

$$
\operatorname{End}_{A}^{g}(A)=\left\{f_{g} \in \operatorname{End}_{A-}(A) \mid f_{g}(a b)=f_{g}(a)(g \cdot b), \text { for all } a, b \in A\right\} .
$$

Consequently, every $f \in \operatorname{Hom}_{A^{\mathrm{e}}}(A, A \# k G)$ can be written uniquely as

$$
f(a)=\sum_{g \in G} f_{g}(a) \# g,
$$


with $f_{g} \in \operatorname{End}_{A}^{g}(A)$, and only finitely many of the $f_{g}$ different from zero.

Proof. Assume that $f \in \operatorname{Hom}_{A^{\mathrm{e}}}(A, A \# k G)$ is homogeneous of degree $g$. Then $f(a) \in A \# k g$, for every $a \in A$, so we can write $f(a)=f_{g}(a) \# g$. From the fact that $f$ is left $A$-linear, it follows that $f_{g}$ is left $A$-linear. From the fact that $f$ is right $A$-linear, we obtain

$$
f_{g}(a)(g \cdot b) \# g=f(a) b=f(a b)=f_{g}(a b) \# g,
$$

and the result follows.

Lemma 2.3. Let $A$ be a $G$-module Taylor-Azumaya algebra. Then we have an isomorphism of algebras

$$
M(A \# k G)^{A} \cong \operatorname{Hom}_{A^{\mathrm{e}}}(A, A \# k G) .
$$

Proof. Take a multiplier $x=\left(\rho_{1}, \rho_{2}\right) \in M(A \# k G)$. Observe that $x \in M(A \# k G)^{A}$ if and only if $\rho_{1}$ is left $A$-linear and $\rho_{2}$ is right $A$-linear. We now define a map

$$
\alpha: M(A \# k G)^{A} \rightarrow \operatorname{Hom}_{A^{\mathrm{e}}}(A, A \# k G), \alpha\left(\rho_{1}, \rho_{2}\right)=f,
$$

with $f(a)=\rho_{2}(a \# 1) . f$ is left $A$-linear since $\left(\rho_{1}, \rho_{2}\right)$ is a multiplier, and from the fact that $\rho_{2}$ is also right $A$-linear, it follows that $f$ is right $A$-linear. Thus $f$ is an $A$-bimodule map and hence a left $A^{e}$-map. Next we define

$$
\beta: \operatorname{Hom}_{A^{\mathrm{e}}}(A, A \# k G) \rightarrow M(A \# k G)^{A}, \beta(f)=\left(\rho_{1}, \rho_{2}\right),
$$

with

$$
\rho_{1}(a \# g)=f(a)(1 \# g) \text { and } \rho_{2}(a \# g)=(1 \# g) f\left(g^{-1} \cdot a\right) .
$$

We remark that $1 \# g \in M(A \# k G)$. $\rho_{1}$ is right $A \# k G$-linear since

$$
\begin{gathered}
\rho_{1}((a \# g)(b \# h))=\rho_{1}(a(g \cdot b) \# g h)=f(a(g \cdot b))(1 \# g h) \\
=f(a)(g \cdot b \# g h)=\rho_{1}(a \# g)(b \# h) .
\end{gathered}
$$

In a similar fashion, we can show that $\rho_{2}$ is left $A$-linear. We next compute that

$$
\begin{aligned}
& (a \# g) \rho_{1}(b \# h)=(a \# g) f(b)(1 \# h)=(1 \# g)\left(g^{-1} \cdot a \# 1\right) f(b)(1 \# h) \\
& \quad=(1 \# g) f\left(\left(g^{-1} \cdot a\right) b\right)(1 \# h)=(1 \# g) f\left(g^{-1} \cdot a\right)(b \# h)=\rho_{2}(a \# g)(b \# h),
\end{aligned}
$$

and it follows that $\left(\rho_{1}, \rho_{2}\right)$ is a multiplier. From the fact that $f$ is left $A$-linear, it follows immediately that $\rho_{1}$ is left $A$-linear. $\rho_{2}$ is right $A$-linear since

$$
\begin{gathered}
\rho_{2}((a \# g) b)=\rho_{2}(a(g \cdot b) \# g)=(1 \# g) f\left(\left(g^{-1} \cdot a\right) b\right) \\
=(1 \# g) f\left(g^{-1} \cdot a\right) b=\rho_{2}(a \# g) b,
\end{gathered}
$$

where we now use the fact that $f$ is right $A$-linear. This shows that $\beta(f)=\left(\rho_{1}, \rho_{2}\right) \in$ $M(A \# k G)^{A}$. It is clear that $\alpha$ is the inverse of $\beta$. Finally, $\alpha$ is an algebra homomorphism. Indeed, if $\alpha\left(\rho_{1}, \rho_{2}\right)=f$ and $\alpha\left(\rho_{1}^{\prime}, \rho_{2}^{\prime}\right)=f^{\prime}$, then

$$
\begin{aligned}
& \alpha\left(\rho_{1} \circ \rho_{1}^{\prime}, \rho_{2}^{\prime} \circ \rho_{2}\right)(a b)=\rho_{2}^{\prime}\left(\rho_{2}(a b \# 1)\right)=\rho_{2}^{\prime}\left(\rho_{2}(a \# 1) b\right) \\
& \quad=\rho_{2}^{\prime}\left(\rho_{2}(a \# 1)(b \# 1)\right)=\rho_{2}(a \# 1) \rho_{2}^{\prime}(b \# 1)=f(a) f^{\prime}(b)=\left(f f^{\prime}\right)(a b),
\end{aligned}
$$

where we use the fact that $\rho_{2}$ is right $A$-linear and $\rho_{2}^{\prime}$ is left $A \# k G$-linear.

Lemma 2.4. We have an algebra anti-homomorphism

$$
p: \operatorname{Hom}_{A^{\mathrm{e}}}(A, A \# k G) \rightarrow M(A) .
$$


Proof. Take $f \in \operatorname{Hom}_{A^{\mathrm{e}}}(A, A \# k G)$, and define $p(f)=\left(\sigma_{1}, \sigma_{2}\right)$ by

$$
\sigma_{1}(a)=\sum_{h \in G} f_{h}\left(h^{-1} \cdot a\right) \text { and } \sigma_{2}(a)=\sum_{h \in G} f_{h}(a),
$$

where we use the notation introduced in Lemma 2.2 It is straightforward to show that $\left(\sigma_{1}, \sigma_{2}\right)$ is a multiplier. Let us show that $p$ is an anti-homomorphism. Take $f, f^{\prime} \in \operatorname{Hom}_{A^{\mathrm{e}}}(A, A \# k G)$, and let $p(f)=\left(\sigma_{1}, \sigma_{2}\right), p\left(f^{\prime}\right)=\left(\sigma_{1}^{\prime}, \sigma_{2}^{\prime}\right), p\left(f f^{\prime}\right)=$ $\left(\tau_{1}, \tau_{2}\right)$. We have to show that $\tau_{2}=\sigma_{2} \circ \sigma_{2}^{\prime}$ and $\tau_{1}=\sigma_{1}^{\prime} \circ \sigma_{1}$. It suffices to prove this for $f$ and $f^{\prime}$ homogeneous, respectively of degree $g$ and $h$. We then have that $f(a)=f_{g}(a) \# g$ and $f^{\prime}(a)=f_{h}^{\prime}(a) \# h$, and we compute

$$
\begin{aligned}
\left(f f^{\prime}\right)(a b) & =\left(f_{g}(a) \# g\right)\left(f_{h}^{\prime}(b) \# h\right)=f_{g}(a)\left(g \cdot f_{h}^{\prime}(b)\right) \# g h \\
& =f_{g}\left(a f_{h}^{\prime}(b)\right) \# g h=f_{g}\left(f_{h}^{\prime}(a b)\right) \# g h .
\end{aligned}
$$

It follows immediately that

$$
\tau_{2}(a b)=f_{g}\left(f_{h}^{\prime}(a b)\right)=\left(\sigma_{2} \circ \sigma_{2}^{\prime}\right)(a b) .
$$

We finally compute

$$
\begin{gathered}
\left(\sigma_{1}^{\prime} \circ \sigma_{1}\right)(a b)=f_{h}^{\prime}\left(h^{-1} \cdot f_{g}\left(g^{-1} \cdot(a b)\right)\right)=f_{h}^{\prime}\left(h^{-1} \cdot\left(\left(g^{-1} \cdot a\right) f_{g}\left(g^{-1} \cdot b\right)\right)\right) \\
\quad=f_{h}^{\prime}\left(\left(\left(h^{-1} g^{-1}\right) \cdot a\right)\left(h^{-1} \cdot f_{g}\left(g^{-1} \cdot b\right)\right)\right)=f_{h}^{\prime}\left((g h)^{-1} \cdot a\right) f_{g}\left(g^{-1} \cdot b\right) \\
=f_{g}\left(f_{h}^{\prime}\left((g h)^{-1} \cdot a\right)\left(g^{-1} \cdot b\right)\right)=f_{g}\left(f_{h}^{\prime}\left((g h)^{-1} \cdot(a b)\right)\right)=\tau_{1}(a b) .
\end{gathered}
$$

Example 2.5. Let $A$ be a Taylor-Azumaya algebra, with trivial $G$-action: $g \cdot a=a$, for all $g \in G$ and $a \in A$. Then $A$ is a $G$-module Taylor-Azumaya algebra, and $A \# k G=A \otimes k G$ as $k$-algebras. Furthermore,

$\operatorname{Hom}_{A^{\mathrm{e}}}(A, A \otimes k G) \cong \operatorname{Hom}_{A^{\mathrm{e}} \otimes k}(A \otimes k, A \otimes k G) \cong \operatorname{Hom}_{A^{\mathrm{e}}}(A, A) \otimes k G \cong k G$.

Recall from 3 that an action by a group $G$ on an algebra $A$ with identity is called strongly inner if there is a group morphism $f: G \rightarrow U(A)$, the group of units of $A$, such that $g \cdot a=f(g) a f\left(g^{-1}\right)$ for all $g \in G$ and $a \in A$. Obviously, this definition makes no sense if $A$ has no unit. We then have the following generalization.

Definition 2.6. Let $G$ be a group and $A$ a unital algebra. An action of $G$ on $A$ is called strongly inner if there exists a group morphism $f: G \rightarrow U(M(A))$, the group of units of $M(A)$, such that $g \cdot a=f(g) a f\left(g^{-1}\right)$, for all $g \in G$ and $a \in A$.

It is easy to see that a strongly inner action of $G$ on an algebra $A$ can be extended to a strongly inner action on the multiplier algebra $M(A)$ of $A$.

Lemma 2.7. Let $\underline{M}=\left(M, M^{\prime}, \mu\right)$ be a dual pair of $G$-modules. Then the induced action of $G$ on $E(\underline{M})$ is strongly inner. Conversely, a strongly inner $G$-action on an elementary algebra $E(\underline{M})$ is induced by a $G$-action on $\underline{M}$.

Proof. Let $\psi: G \rightarrow \operatorname{End}(M)$ and $\psi^{\prime}: G \rightarrow \operatorname{End}\left(M^{\prime}\right)$ be the representation maps of $G$ on $M$ and $M^{\prime}$. Then $\left(\psi(g), \psi^{\prime}\left(g^{-1}\right)\right) \in \bar{E}$ (see Proposition 1.3) since

$$
\mu\left(m \otimes g^{-1} \cdot m^{\prime}\right)=\mu\left(g \cdot m \otimes\left(g g^{-1}\right) \cdot m^{\prime}\right)=\mu\left(g \cdot m \otimes m^{\prime}\right) .
$$


Let $f(g)$ be the multiplier corresponding to $\left(\psi(g), \psi^{\prime}\left(g^{-1}\right)\right)$, as in Proposition 1.3 . Then we compute

$$
g \cdot\left(m \otimes m^{\prime}\right)=\psi(g)(m) \otimes \psi^{\prime}(g)\left(m^{\prime}\right)=f(g)\left(m \otimes m^{\prime}\right) f\left(g^{-1}\right) .
$$

Conversely, assume that $G$ acts strongly innerly on an elementary algebra $E$. Then there exists a group morphism $\lambda: G \rightarrow U(M(E)) \cong U(\bar{E}), \lambda(g)=\left(f(g), f^{\prime}(g)\right) \in$ $\bar{E}$ such that

$$
g \cdot\left(m \otimes m^{\prime}\right)=\lambda(g)\left(m \otimes m^{\prime}\right) \lambda\left(g^{-1}\right)=f_{g}(m) \otimes f_{g^{-1}}^{\prime}\left(m^{\prime}\right) .
$$

Now define actions of $G$ on $M$ and $M^{\prime}$ by $g \cdot m=f_{g}(m)$ and $g \cdot m^{\prime}=f_{g}^{\prime}\left(m^{\prime}\right)$. These actions are associative because $F$ is a group homomorphism. Using the fact that $\left(f(g), f^{\prime}(g)\right) \in \bar{E}$, we compute

$$
\mu\left(g \cdot m^{\prime} \otimes g \cdot m\right)=\mu\left(f_{g^{-1}}^{\prime}\left(m^{\prime}\right) \otimes f_{g}(m)\right)=\mu\left(m^{\prime} \otimes f_{g^{-1}}^{\prime}\left(f_{g}(m)\right)\right)=\mu\left(m^{\prime} \otimes m\right),
$$

so $\underline{M}$ is a dual pair of $G$-modules.

Lemma 2.8. Let $A$ be a $G$-module Taylor-Azumaya algebra. The G-action on $A$ is strongly inner if and only if $\operatorname{Hom}_{A^{\mathrm{e}}}(A, A \# k G) \cong k G$ as $G$-graded algebras.

Proof. First suppose that the $G$-action is strongly inner. There exists $f: G \rightarrow$ $M(A)$ such that $g \cdot a=f(g) a f\left(g^{-1}\right)$, for all $g \in G$ and $a \in A$. The map

$$
\alpha: A \otimes k G \rightarrow A \# k G, \alpha(a \otimes g)=a f\left(g^{-1}\right) \# g
$$

is an isomorphism of $G$-graded unital $A^{e}$-modules. Consequently, $H_{l}(A \otimes k G) \cong$ $H_{l}(A \# k G)$ as $G$-graded modules, and it is easy to see that this is a morphism of $G$-graded algebras. Now we have seen in Example 2.5 that $H_{l}(A \otimes k G)=k G$, and one implication follows.

Conversely, assume that there exists an isomorphism of graded algebras $\eta$ : $k G \rightarrow \operatorname{Hom}_{A^{\mathrm{e}}}(A, A \# k G)$. The composition with the map $p$ introduced in Lemma 2.4 is an algebra anti-homomorphism $p \circ \eta: k G \rightarrow M(A)$. Therefore, the map

$$
f: G \rightarrow U(M(A)), f(g)=p\left(\eta\left(g^{-1}\right)\right)=:\left(\rho_{1, g}, \rho_{2, g}\right)
$$

is a group homomorphism. According to the computations in the proof of Lemma 2.4. and using the fact that $\eta(g)$ is homogenous of degree $g$, we have

$$
f(g) a=\rho_{1, g}(a)=\eta\left(g^{-1}\right)(g \cdot a) \text { and } a f\left(g^{-1}\right)=\rho_{2, g^{-1}}(a)=\eta(g),
$$

and it follows that

$$
f(g) a f\left(g^{-1}\right)=\left(\eta(g) \circ \eta\left(g^{-1}\right)\right)(g \cdot a)=g \cdot a,
$$

as needed.

Lemma 2.9. Let $A$ and $B$ be $G$-module Taylor-Azumaya algebras. Then we have an isomorphism of graded algebras

$$
\begin{aligned}
\alpha: \operatorname{Hom}_{A^{\mathrm{e}}}(A, A \# k G) \square \operatorname{Hom}_{B}^{\mathrm{e}}(B, B \# k G) \\
\rightarrow \operatorname{Hom}_{(A \otimes B)^{\mathrm{e}}}((A \otimes B),(A \otimes B) \# k G) .
\end{aligned}
$$

Proof. The part of degree $g$ of the cotensor product on the left-hand side is generated by $f \# \tilde{f}$, with $f: A \rightarrow A \# k G$ and $\tilde{f}: B \rightarrow B \# k G$ homogeneous of degree $g$. So we have $f(a)=f_{g}(a) \# g, \tilde{f}(b)=\tilde{f}_{g}(b) \# g$, for all $a \in A$ and $b \in B$ (see Lemma 2.2). Now we define $\alpha(f \otimes \tilde{f})$ by

$$
\alpha(f \otimes \tilde{f})(a \otimes b)=\left(f_{g}(a) \otimes \tilde{f}_{g}(b)\right) \# g .
$$


It is clear that $\alpha(f \otimes \tilde{f})$ is a morphism of left $A^{\mathrm{e}}$-modules of degree $g$, and $\alpha$ is a morphism of graded $k$-algebras. Any morphism of graded $k$-algebras between $k G$ Galois objects is an isomorphism (see [2, Prop. 8.1.10]), hence $\alpha$ is an isomorphism.

Collecting the results of all the previous lemmas, we obtain:

Corollary 2.10. We have a well-defined group homomorphism $\pi: \operatorname{BM}^{\prime}(k, G) \rightarrow$ $\operatorname{Gal}(k, G)$, given by $\pi[A]=\left[\operatorname{Hom}_{A^{\mathrm{e}}}(A, A \# k G)\right]$. $\operatorname{Ker}(\pi)$ contains $\operatorname{Br}^{\prime}(k)$.

Let $B$ be a $k G$-Galois object. The inverse map $\beta^{-1}$ of the canonical isomorphism $\beta: B \otimes B \rightarrow B \otimes k G$ induces the Miyashita action of $G$ on $B$ (see [13):

$$
g \neg b=\sum X_{i}^{g} b Y_{i}^{g}, \quad \sum X_{i}^{g} \otimes Y_{i}^{g}=\beta^{-1}(1 \otimes g) .
$$

The $G$-graded module $B$ with the Miyashita $G$-action (3) is a Yetter-Drinfeld module, that is, the $G$-action and the $G$-grading satisfy the compatibility relation $g \rightarrow b_{\sigma} \in B_{g \sigma g^{-1}}$, for every homogeneous element $b_{\sigma} \in B_{\sigma}$ and $g \in G$. Moreover, $B$ is quantum-commutative in the sense that

$$
b_{\sigma} a=(\sigma \neg a) b_{\sigma}
$$

for all $a \in B, b_{\sigma} \in B_{\sigma}$. For the detail on Yetter-Drinfeld modules and quantumcommutative algebras, the reader may refer to [5, 6].

Lemma 2.11. The group homomorphism $\pi$ is split surjective.

Proof. Let $B$ be a $k G$-Galois object, and consider the multiplier Hopf algebra $k(G)$ from Example 1.4. We show that $A=B \# k(G)$ is a $G$-Taylor-Azumaya algebra and $\pi(A) \cong B$. Since $A$ is clearly flat, $A$ will be equivalent to some finitely generated G-Taylor-Azumaya algebra. By [18, Thm.4.3], there exists a strict Morita context

$$
\{B \# k(G), k, B, B,[,],(,)\}
$$

where $[a, b]=a p_{e} b=\sum a\left(p_{g} \cdot b\right) \# p_{g^{-1}}$ and $(a, b)=(a b)_{e}$ for all $a, b \in B$, and $e$ is the identity of $G$. It follows that $A$ is isomorphic to the elementary Taylor-Azumaya algebra $E(\underline{B})$, where $\underline{B}=(B, B,[]$,$) . So A$ is a $G$-module Taylor-Azumaya algebra. Define $\phi: B \rightarrow \pi(A)$ by

$$
\phi\left(b_{\sigma}\right)=\sum_{g \in G}\left(\left(g \rightarrow b_{\sigma}\right) \# p_{g}\right) \# \sigma \in M(A \# k G)
$$

for all homogeneous $b_{\sigma} \in B_{\sigma}$. It is obvious that $\phi$ is graded. We show that $\phi$ is a well-defined algebra map. First we verify that $\phi(B) \subseteq \pi(A)$. Let $x=a_{\delta} \# p_{h} \in$ $A=B \# k(G)$ and $b_{\sigma} \in B_{\sigma}$. Identify $x$ with $x \# 1$ in $A \# k G$. Then we have

$$
\begin{aligned}
& \phi\left(b_{\sigma}\right)(x \# 1)=\phi\left(b_{\sigma}\right)\left[\left(a_{\delta} \# p_{h}\right) \# 1\right] \\
& \quad=\sum_{g \in G}\left[\left(\left(g \neg b_{\sigma}\right) \# p_{g}\right) \# \sigma\right]\left[\left(a_{\delta} \# p_{h}\right) \# 1\right]=\sum_{g \in G}\left[\left(\left(g \neg b_{\sigma}\right) \# p_{g}\right)\left(a_{\delta} \# p_{h \sigma^{-1}}\right)\right] \# \sigma \\
& =\sum_{g \in G}\left[\left(g \neg b_{\sigma}\right)\left(p_{g(1)} \cdot a_{\delta}\right) \# p_{g(2)} p_{h \sigma^{-1}}\right] \# \sigma=\sum_{g \in G}\left[\left(g \neg b_{\sigma}\right) a_{\delta} \# p_{\delta^{-1} g} p_{h \sigma^{-1}}\right] \# \sigma \\
& =\left[\left(\delta h \sigma^{-1} \rightarrow b_{\sigma}\right) a_{\delta} \# p_{h \sigma^{-1}}\right] \# \sigma=\left[a_{\delta}\left(h \sigma^{-1} \neg b_{\sigma}\right) \# p_{h \sigma^{-1}}\right] \# \sigma,
\end{aligned}
$$


by (44). On the other hand, we have $g \rightarrow b_{\sigma} \in B_{g \sigma g^{-1}}$ and

$$
\begin{aligned}
(x & \# 1) \phi\left(b_{\sigma}\right)=\sum_{g \in G}\left[\left(a_{\delta} \# p_{h}\right) \# 1\right]\left[\left(\left(g \neg b_{\sigma}\right) \# p_{g}\right) \# \sigma\right] \\
& =\sum_{g \in G}\left[\left(a_{\delta} \# p_{h}\right)\left(\left(g \neg b_{\sigma}\right) \# p_{g}\right)\right] \# \sigma \\
& =\sum_{g \in G}\left[a_{\delta}\left(p_{h(1)} \cdot\left(g \neg b_{\sigma}\right)\right) \# p_{h(2)} p_{g}\right] \# \sigma \\
& =\sum_{g \in G}\left[a_{\delta}\left(g \neg b_{\sigma}\right) \# p_{g \sigma^{-1} g^{-1} h} p_{g}\right] \# \sigma \\
& =\left[a_{\delta}\left(h \sigma^{-1} \rightarrow b_{\sigma}\right) \# p_{h \sigma^{-1}}\right] \# \sigma .
\end{aligned}
$$

Thus we have $(x \# 1) \phi(b)=\phi(b)(x \# 1)$ for all $x \in A$ and $b \in B$. So $\phi(B) \subseteq \pi(A)$. Next we show that $\phi$ is an algebra map. Let $a_{\sigma} \in B_{\sigma}$ and $b_{\delta} \in B_{\delta}$. Then

$$
\begin{aligned}
\phi & \left(a_{\sigma}\right) \phi\left(b_{\delta}\right)=\left[\sum_{g \in G}\left(\left(g \neg a_{\sigma}\right) \# p_{g}\right) \# \sigma\right]\left[\sum_{h \in G}\left(\left(h \neg b_{\delta}\right) \# p_{h}\right) \# \delta\right] \\
& =\sum_{g, h \in G}\left[\left(g \neg a_{\sigma}\right) \# p_{g}\right]\left[\left(h \neg b_{\delta}\right) \# p_{h \sigma^{-1}}\right] \# \sigma \delta \\
& =\sum_{g, h \in G}\left[\left(g \neg a_{\sigma}\right)\left(p_{g_{(1)}} \cdot\left(h \neg b_{\delta}\right)\right) \# p_{g_{(2)}} p_{h \sigma^{-1}}\right] \# \sigma \delta \\
& =\sum_{g, h \in G}\left[\left(g \neg a_{\sigma}\right)\left(h \neg b_{\delta}\right) \# p_{\left(h \delta h^{-1}\right)^{-1} g} p_{h \sigma^{-1}}\right] \# \sigma \delta \\
& =\sum_{h \in G}\left[\left(h \delta \sigma^{-1} \neg a_{\sigma}\right)\left(h \neg b_{\delta}\right) \# p_{h \sigma^{-1}}\right] \# \sigma \delta \\
& =\sum_{t \in G}\left[\left(t \delta^{\sigma} \neg a_{\sigma}\right)\left(t \sigma \neg b_{\delta}\right) \# p_{t}\right] \# \sigma \delta \\
& =\sum_{t \in G}\left[t \neg\left(\left(\delta^{\sigma} \neg a_{\sigma}\right)\left(\sigma \neg b_{\delta}\right)\right) \# p_{t}\right] \# \sigma \delta \\
& =\sum_{t \in G}\left[t \neg\left(\left(\sigma \neg b_{\delta}\right) a_{\sigma}\right) \# p_{t}\right] \# \sigma \delta \\
& =\sum_{t \in G}\left[t \neg\left(a_{\sigma} b_{\delta}\right) \# p_{t}\right] \# \sigma \delta=\phi\left(a_{\sigma} b_{\delta}\right),
\end{aligned}
$$

where $\delta^{\sigma}=\sigma \delta \sigma^{-1}$. In the eighth and ninth equality, we used the quantum-commutativity (4) of $B$. Finally, $\phi$ is an isomorphism since $B$ and $\pi(A)$ are $k G$-Galois objects.

We can now prove the main result of this paper.

Theorem 2.12. Let $G$ be a group and $k$ a commutative ring. Then we have the following split exact sequence

$$
1 \rightarrow \operatorname{Br}^{\prime}(k) \rightarrow \mathrm{BM}^{\prime}(k, G) \stackrel{\pi}{\longrightarrow} \operatorname{Gal}(k, G) \rightarrow 1 .
$$

Proof. By Lemma 2.11 it suffices to show that $\operatorname{Ker}(\pi)=\operatorname{Br}^{\prime}(k)$. Let $\iota: \operatorname{Br}^{\prime}(k) \rightarrow$ $\operatorname{BM}^{\prime}(k, G)$ be the canonical embedding. We define a group morphism $\zeta: \operatorname{Ker}(\pi) \rightarrow$ $\operatorname{Br}^{\prime}(k)$ by forgetting the $G$-action on $G$-module Taylor-Azumaya algebras. It is easy to see that $\zeta \circ \iota$ is the identity automorphism of $\mathrm{Br}^{\prime}(k)$. Thus $\zeta$ is a surjective map. 
We show that $\zeta$ is injective as well. Let $A$ be a $G$-module Taylor-Azumaya algebra such that the underlying algebra $A$ is elementary, say $A \cong E(\underline{M})$ for some dual pair of $k$-modules $\underline{M}=\left(M, M^{\prime}, \mu\right)$. Since $\pi(A) \cong k G$, it follows from Lemma 2.8 that the $G$-actions on $A$ and on $E(\underline{M})$ are strongly inner. By Lemma 2.7, there exists a $G$-module structure on $\underline{M}$ that induces the $G$-module structure on $E(\underline{M})$, hence $[E(\underline{M})]=1$.

If $k$ is a field, $\operatorname{Br}^{\prime}(k)=\operatorname{Br}(k), \operatorname{Gal}(k, G)=H^{2}\left(G, k^{*}\right)$, and $\mathrm{BM}^{\prime}(k, G)=\operatorname{BM}(k, G)$, and the exact sequence (5) is isomorphic to Fröhlich's exact sequence 8 , Theorem 4.1]. If $G$ is finite, then the Picco-Platzeck exact sequence (cf. [14]) is a "subsequence" of (5). In this case, $\pi$ restricts to a surjective map on $\operatorname{BM}(k, G)$ and the kernel of the restricted map $\pi$ is $\operatorname{Br}(k)$.

It is not hard to generalize the exact sequence (5) to the equivariant Brauer group of an infinite cocommutative Hopf algebra with an integral, which will yield the infinite version of Beattie's exact sequence [1. The generalization to the equivariant Brauer group of an infinite quantum group seems far from obvious.

\section{REFERENCES}

[1] M. Beattie, A direct sum decomposition for the Brauer group of $H$-module algebras, $J$. Algebra 43 (1976), 686-693. MR0441942 (56:333)

[2] S. Caenepeel, "Brauer groups, Hopf algebras and Galois theory", K-Monographs in Mathematics 4, Kluwer Academic Publishers, Dordrecht, 1998. MR.1610222 (99b:16028)

[3] R. Blattner, M. Cohen, and S. Montgomery, Crossed products and inner actions of Hopf algebras, Trans. Amer. Math. Soc. 298 (1986), 672-711. MR0860387 (87k:16012)

[4] S. Caenepeel and F. Grandjean, A note on Taylor's Brauer group, Pacific J. Math 186 (1998), 13-27. MR1665054 (2000f:14027)

[5] S. Caenepeel, F. Van Oystaeyen and Y.H. Zhang, Quantum Yang-Baxter module algebras, K-Theory, 8(1993), 231-255. MR1291020(95e:16031)

[6] S. Caenepeel, F. Van Oystaeyen and Y.H. Zhang, The Brauer group of Yetter-Drin'feld module algebras, Trans. Amer. Math. Soc. 349 (1997), 3737-3771. MR1454120 (98c:16047)

[7] L.N. Childs, The Brauer group of graded Azumaya algebras II: graded Galois extensions, Trans. Amer. Math. Soc. 204 (1975), 137-160. MR0364216 (51:471)

[8] A. Fröhlich, Orthogonal and symplectic representations of groups, Proc. London Math. Soc. (3) 24 (1971), 470-506. MR0308248 (46:7362)

[9] A. Fröhlich, C.T.C. Wall, Equivariant Brauer groups in algebraic number theory, Bull. Soc. Math. France 25 (1971), 91-96. MR1803361 (2001m:18008)

[10] A. Fröhlich, C.T.C. Wall, Equivariant Brauer groups, Contemp. Math. 272 (2000), 57-71. MR.1803361 (2001m:18008)

[11] O. Gabber, Some theorems on Azumaya algebras, in "Groupe de Brauer", M. Kervaire and M. Ojanguren (Eds.), Lecture Notes Math. 844, Springer-Verlag, Berlin, 1981. MR0611868 (83d:13004)

[12] M.A. Knus, M. Ojanguren, Cohomologie étale et groupe de Brauer, in "Groupe de Brauer", M. Kervaire and M. Ojanguren (Eds.), Lecture Notes in Math. 844, Springer-Verlag, Berlin, 1981. MR0611869 (83k:16006)

[13] Y. Miyashita, An exact sequence associated with a generalized crossed product, Nagoya Math. J. 49 (1973), 21-51. MR0320080 (47:8621)

[14] D.J. Picco, M.I. Platzeck, Graded algebras and Galois extensions, Bol. Un. Mat. Argentina 25 (1971), 401-415. MR0332894 (48:11219)

[15] I. Raeburn, J. L. Taylor, The bigger Brauer group and étale cohomology, Pacific J. Math. 119 (1985), 445-463. MR0803128(87a:16007)

[16] J.L. Taylor, A bigger Brauer group, Pacific J. Math. 103 (1982), 163-203. MR0687968 (84g:13007)

[17] A. Van Daele, Multiplier Hopf algebras, Trans. Amer. Math. Soc. 342 (1994), 917-932. MR:1220906 (94h:16075) 
[18] A. Van Daele, Y.H. Zhang, Galois theory for multiplier Hopf algebras with integrals, Algebras Representation Theory, 2 (1999), 83-106. MR1688472 (2000f:16050)

[19] A. Van Daele, Y.H. Zhang, A survey on multiplier Hopf algebras, in "Hopf algebras and quantum groups", S. Caenepeel and F. Van Oystaeyen (eds.), Lecture Notes Pure Appl. Math. 209, Marcel Dekker, New York, 2000, 269-309. MR767620(2001e:16077)

Faculty of Applied Sciences, Vrije Universiteit Brussel, VUB, B-1050 Brussels, BelGIUM

E-mail address: scaenepe@vub.ac.be

URL: http://homepages.vub.ac.be/ ${ }^{\text {scaenepe/ }}$

Department of Mathematics and Computer Science, University of Antwerp, MiddelheimlaAn 1, B-2020 Antwerp, Belgium

E-mail address: fred.vanoystaeyen@ua.ac.be

School of Mathematics and Computing Science, Victoria University of Wellington, Wellington, New ZeALAND

E-mail address: yinhuo.zhang@vuw.ac.nz 